\title{
Classical anisotropies in models of open inflation
}

\author{
Jaume Garriga \\ IFAE, Departament de Física, Universitat Autònoma de Barcelona, 08193 Bellaterra, Barcelona, Spain \\ and Center For Theoretical Physics, Laboratory for Nuclear Science and Department of Physics, Massachusetts Institute of Technology, \\ Cambridge, Massachusetts 02139 \\ V. F. Mukhanov \\ Institut für Theoretische Physik, ETH Zürich, CH-8093 Zürich, Switzerland
}

(Received 6 March 1997)

\begin{abstract}
In the simplest model of open inflation there are two inflaton fields decoupled from each other. One of them, the tunneling field, produces a first stage of inflation which prepares the ground for the nucleation of a highly symmetric bubble. The other, a free field, drives a second period of slow-roll inflation inside the bubble. However, the second field also evolves during the first stage of inflation, which to some extent breaks the needed symmetry. We show that this generates large supercurvature anisotropies which, together with the results of Tanaka and Sasaki, rule out this class of simple models (unless, of course, $\Omega_{0}$ is sufficiently close to 1). The problem does not arise in modified models where the second field does not evolve in the first stage of inflation. [S0556-2821(97)01216-2]

PACS number(s): $98.80 . \mathrm{Cq}$
\end{abstract}

Open inflation has recently received some attention as a viable model for the early universe [1-5]. The first version of the open universe model was based on the theory of one scalar field $[1,2]$. However, no realistic models of that type have been proposed so far. Then Linde and Mezhlumian proposed a class of models involving two scalar fields [3]. The simplest model discussed in [3] describes a tunneling field $\sigma$, responsible for bubble nucleation, and a free field $\phi$ of mass $m$ that undergoes slow rollover. The two fields are decoupled from each other, except of course gravitationally. When the field $\sigma$ is in its false vacuum, it dominates the energy density and the universe is in a de Sitter phase with constant Hubble rate $H_{1}$. The true vacuum of the the field $\sigma$ has vanishing energy density, and when a bubble nucleates, the slowly rolling field $\phi$ drives a second period of inflation in its interior, with Hubble rate $H_{2}=\left(m^{2} \phi^{2}\right)^{1 / 2}$.

In order for this model to work, the field $\phi$ has to evolve very slowly outside the bubble. Otherwise, the surfaces of constant $\phi$ would not be well synchronized with the hyperboloids of constant $\sigma$ inside the bubble, and large anisotropies could be expected. The danger of this effect was already realized in [3], therefore Linde and Mezhlumian also suggested the possible modifications of the simplest model where synchronization was exact and no such problems appear. However, since the simplest model looks more natural, it would still be interesting to clarify whether or not it is compatible with observations for some range of the parameters.

Outside the bubble of the field $\sigma$, we have

$$
\phi(\mathbf{x}, t) \approx \phi_{s} \equiv A e^{-\alpha H_{1} \hat{t}}
$$

where $\hat{t}$ is the cosmological time in the flat FriedmannRobertson-Walker (FRW) chart, and $\alpha=m^{2} / 3 H_{1}^{2} \ll 1$ is the slow-rollover parameter. We shall now concentrate on fluc- tuations which will arise because of the $\hat{t}$ dependence of $\phi$, so we take $A$ to be constant over the region of interest.

The true and false vacua for the field $\sigma$ are strongly nondegenerate, which means we are in the thick wall regime. For simplicity, we shall take the solution (1) to be valid everywhere outside the forward light cone from the nucleation event. This approximation is clearly valid if the bubble size at the moment of nucleation is small compared with $H_{1}^{-1}$.

Inside the light cone, the spacetime can be covered with the open chart

$$
d s^{2}=-d t^{2}+a^{2}(t)\left[d r^{2}+\sinh ^{2} r\left(d \theta^{2}+\sin ^{2} \theta d \varphi^{2}\right)\right] .
$$

The scale factor $a$ obeys the Friedmann equation

$$
(1-\Omega) \dot{a}^{2}=1,
$$

where $\Omega$ is the density parameter and at early times $a(t) \approx t$. To propagate the solution (1) to the inside of the light cone we can use the following trick. Let us assume that inside the bubble, and for $t<t_{*} \ll H_{1}^{-1}$, the universe is still de Sitter, with the same Hubble parameter $H_{1}$. Since at early times the universe is dominated by curvature, this assumption will turn out to be harmless. Introducing in Eq. (1) the relation between the flat and open de Sitter coordinates $\hat{t}=\ln \left(\cosh H_{1} t+\sinh H_{1} t \cosh r\right)$, we have

$$
\phi_{s}=A\left(\cosh H_{1} t+\sinh H_{1} t \cosh r\right)^{-\alpha} .
$$

Of course Eq. (4) satisfies the field equation to first order in the slow-rollover parameter $\alpha$ :

$$
\ddot{\phi}_{s}+3 \frac{\dot{a}}{a} \dot{\phi}_{s}+\frac{\left(\partial_{r}^{2}+2 \operatorname{coth} r \partial r\right)}{a^{2}} \phi_{s}+m^{2} \phi_{s}=O\left(\alpha m^{2} \phi_{s}\right),
$$


with $a=H_{1}^{-1} \sinh \left(H_{1} t\right)$. However, we should note that from the point of view of the open chart, Eq. (4) is not a slowrollover solution. The largest terms in Eq. (5) for $t \ll H_{1}^{-1}$ are the gradient and friction terms, which balance each other.

The question is now to evolve the approximate solution

$$
\phi_{s} \approx\left(1+H_{1} t \cosh r\right)^{-\alpha},
$$

which is valid for $t<t_{*} \ll H_{1}^{-1}$, into the second period of inflation. Since Eq. (6) is not an eigenfunction of the Laplacian, one might be tempted to expand it treating $H_{1} t_{*} \cosh r$ as a small quantity, given that $t_{*}$ can be chosen as small as desired. However, this would not be appropriate. The scale factor behaves as $a(t) \approx t$ up to a time $t \sim H_{2}^{-1}$, where $H_{2} \ll H_{1}$ is the Hubble rate at the beginning of the second period of inflation. Therefore, the comoving size of our causal past (i.e., the region that has an influence on our observable universe) at time $t_{*}$ is

$$
\Delta r \sim-\ln \left(H_{2} t_{*}\right) \gg 1
$$

and $\Delta\left(H_{1} t_{*} \cosh r\right) \sim H_{1} / H_{2} \gg 1$. Hence the second term in the parentheses in Eq. (6) is not uniformly small compared to the first over a sufficiently large patch.

A better strategy is to study a region where the second term in Eq. (6) dominates. Note that since Eq. (6) is spherically symmetric, the universe would still look isotropic around us if we lived in the privileged position $r=0$. The most interesting effects will occur if we live at some $r=r_{0} \gg 1$. Actually, since the volume grows exponentially with $r$, that is where we are most likely to be found. Taking $r_{0}>-\ln \left(\alpha t_{*}^{2} H_{1} H_{2}\right)$, we have

$$
\phi_{s} \approx A\left(1+H_{1} t_{*} \cosh r\right)^{-\alpha} \approx A\left(H_{1} t_{*} \cosh r\right)^{-\alpha}\left[1+O\left(\alpha^{2}\right)\right]
$$

over a region of comoving size (7) centered around $r_{0}$.

It is now convenient to change the coordinates $(r, \theta, \phi)$ on the spacelike hyperboloid to a new set $\left(r^{\prime}, \theta^{\prime}, \phi^{\prime}\right)$ such that the point $r=r_{0}$ will be the new origin of coordinates $r^{\prime}=0$. One can show that

$$
\cosh r=\sinh r_{0} \sinh r^{\prime} \cos \theta^{\prime}+\cosh r_{0} \cosh r^{\prime} .
$$

Our solution is now of the form

$$
\phi_{s} \approx B(t) f^{-\alpha}\left(r^{\prime}, \theta^{\prime}\right),
$$

where

$$
f\left(r^{\prime}, \theta^{\prime}\right) \equiv\left(\cosh r^{\prime}+\sinh r^{\prime} \cos \theta^{\prime}\right) .
$$

Note that, to first order in $\alpha, f^{-\alpha}$ is an approximate eigenfunction of the Laplacian (with eigenvalue $-2 \alpha$ ), and therefore we can factorize the time dependence in Eq. (8).

It can be checked that between the times $t_{*}$ and $t_{2} \sim H_{2}^{-1}$ the gravitational backreaction of the scalar field perturbations can be neglected, and hence all that will happen is that $B(t)$ will evolve as $t^{-\alpha}$. After $t_{2} \sim H_{2}^{-1}$ slow rollover will set in and gravitational backreaction has to be taken into account. For this purpose, one has to separate the field into a background part $\phi_{0}(t)$ and a perturbation $\delta \phi$. Since the gradient terms are negligible in the slow rollover regime (the wavelength of the perturbations we consider is

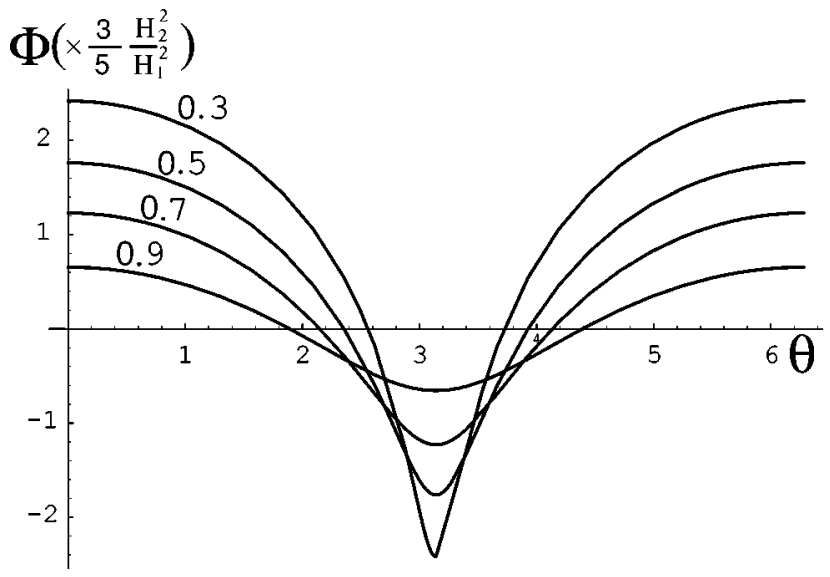

FIG. 1. The gauge potential $\Phi$ at the surface of last scattering $r_{\mathrm{LS}}=\operatorname{arccosh}\left[\left(2 / \Omega_{0}\right)-1\right]$ as a function of the polar angle $\theta^{\prime}$, for $\Omega_{0}$ ranging from 0.3 to 0.9 . (The anisotropy has azimutal symmetry.)

much larger than the horizon), $\delta \phi$ need not be an eigenfunction of the Laplacian, and the decomposition into background and perturbation has some degree of arbitrariness. We choose $\phi_{0}=B\left(t_{2}\right)$ and $\delta \phi=B\left(t_{2}\right) \alpha \ln f$ at the time when the slow rollover starts inside the bubble. The standard procedure then yields the answer that at the end of inflation the gauge-invariant potential is given by

$$
\left.\Phi \approx \frac{3}{5} \frac{H_{2} \delta \phi}{\dot{\phi}_{0}}\right|_{t=t_{2}}=\frac{3}{5}\left(\frac{H_{2}}{H_{1}}\right)^{2} \ln f\left(r^{\prime}, \theta^{\prime}\right) .
$$

Somewhat surprisingly, the answer does not depend on $\mathrm{m}^{2}$ but only on the ratio between the Hubble rates inside and outside the bubble (of course, in a slow-roll model, $\mathrm{H}_{2}$ indirectly depends on $\mathrm{m}^{2}$, for fixed spatial curvature). In Fig. 1 we plot the potential $\Phi$ at the surface of last scattering $r_{\mathrm{LS}}=\operatorname{arccosh}\left[\left(2 / \Omega_{0}\right)-1\right]$ for different values of $\Omega$.

It remains to be seen how $\Phi$ contributes to observables. The present Hubble radius $H_{0}^{-1}$ corresponds to the comoving distance $r_{0}^{\prime}=\left[a\left(t_{0}\right) H_{0}\right]^{-1}=\left(1-\Omega_{0}\right)^{1 / 2}$, where the subindex zero indicates the present time, and we have used Eq. (3). If the present spatial curvature is small, then $r_{0}^{\prime} \ll 1$ and $\ln f$ can be expanded as

$$
\ln f \approx x-\frac{x^{2}}{2}+\frac{x^{3}}{3}+\cdots
$$

where

$$
x \equiv\left(\sinh r^{\prime} \cos \theta^{\prime}+\cosh r^{\prime}\right)-1 \ll 1 .
$$

As mentioned before, $\ln f$ is not an eigenfunction of the Laplacian, and is not normalizable, another illustration of how non-normalizable supercurvature modes can arise in the open inflationary models.

The dominant contribution for small curvature is proportional to $x$. The term in parentheses in Eq. (11) is a supercurvature mode with eigenvalue of the Laplacian equal to +3 , and it can actually be checked that it is pure gauge. It corresponds to the shift $\left(\delta t, \delta x^{i}\right)=\left(\Phi t, \Phi,{ }_{i} \ln t\right)$ during the curvature dominated period. The constant -1 in Eq. (11) 


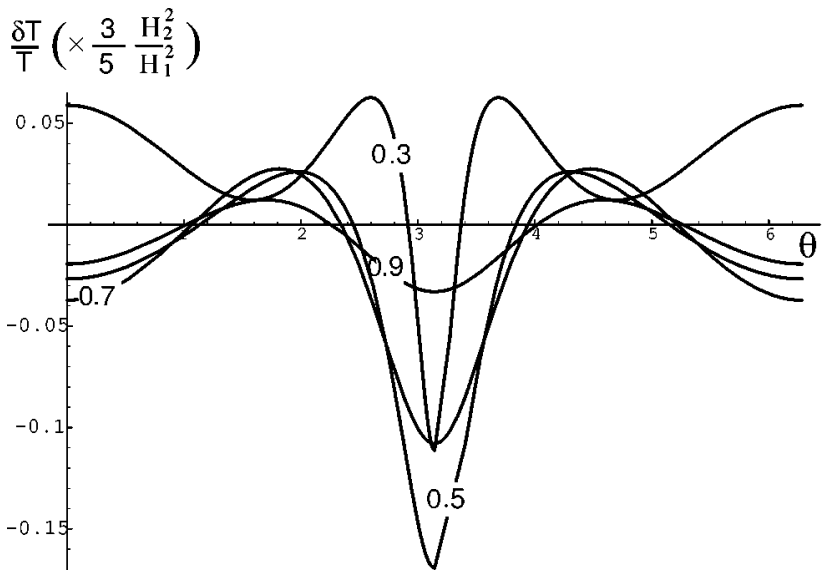

FIG. 2. The temperature anisotropy as a function of the polar angle $\theta^{\prime}$, for the same values of $\Omega_{0}$ as in Fig. 1. This is found from numerical integration of the Sachs-Wolfe effect along the line of sight. The monopole and dipole have been subtracted.

will also not induce any anisotropies. Therefore, the first nontrivial contribution will stem from the $x^{2}$ term in the expansion of $\ln f$. This is proportional to $r^{\prime 2} \cos ^{2} \theta^{\prime}$. Hence, the dominant effect will be in the quadrupole. Higher multipoles, labeled by $l$, will be accompanied by $r^{\prime l}$. Since $r_{\mathrm{LS}}^{\prime} \approx 2\left(1-\Omega_{0}\right)^{1 / 2}$, we have

$$
\left.\frac{\delta T}{T}\right|_{l} ^{\text {class }} \sim f_{l}\left(1-\Omega_{0}\right)^{l / 2}\left(\frac{H_{2}}{H_{1}}\right)^{2},
$$

where $f_{l} \sim 10^{-1}$. We have numerically checked that the above estimate works well by order of magnitude, even for $\Omega_{0}$ as low as 0.3 and $l \lesssim 10$. Notice that this is a classical effect, and cosmic variance cannot be invoked to minimize it. The integrated Sachs-Wolfe effect is shown in Fig. 2 as a function of the polar angle $\theta^{\prime}$ and for different values of
$\Omega_{0}$ (the monopole and dipole contributions have been subtracted). For low $\Omega_{0}$, the anisotropy would show up as a cold spot of small angular size.

Unless the curvature of the universe is very small, the ratio $H_{1} / H_{2}$ would have to be large in order to avoid conflict with observations. For moderate curvature, we need $\left(H_{1} / H_{2}\right) \geq 10^{2}$, whereas for $\left(1-\Omega_{0}\right) \ll 1$ one can get away with a lower ratio.

However, Tanaka and Sasaki have shown that the quantum fluctuations of $\phi$ generated during the first stage of inflation induce an enhancement of the temperature anisotropies in supercurvature modes compared with the fluctuations in subcurvature modes $[6,7]$ :

$$
\left.\frac{\delta T}{T}\right|_{l} ^{\text {sup }} \sim\left(1-\Omega_{0}\right)^{l / 2}\left(\frac{H_{1}}{H_{2}}\right) \frac{\delta T}{T} \text { sub } .
$$

Comparing with Eq. (12) the ratio of Hubble constants appears reversed. If what we observe is due to quantum fluctuations generated during inflation, then $\delta T /\left.T\right|_{\mathrm{sub}} \sim 10^{-5}$. With $\left(H_{1} / H_{2}\right) \geq 10^{2}$, as required above for moderate curvature, the model would give rise to temperature anisotropies of order $10^{-3}$, which are ruled out by observations. Of course the model can be saved if the curvature is sufficiently small, say, $\left(1-\Omega_{0}\right) \lesssim 10^{-2}$.

Also, as we already mentioned, there are alternative models $[3,5]$ in which the slow-roll field $\phi$ does not evolve during the first stage of inflation. These would not be constrained by the mechanism discussed above.

We would like to thank T. Tanaka and A. Linde for useful comments. This work was partially supported by SNF, by the U.S. Department of Energy (DOE) under cooperative research agreement No. DE-FC02-94ER40818, and by CICYT under Project No. AEN95-0882. V.F.M. thanks the Tomalla foundation for financial support.
[1] J. R. Gott, Nature (London) 295, 304 (1982); J. R. Gott and T. S. Statler, Phys. Lett. 136B, 157 (1984); A. Guth and E. Weinberg, Nucl. Phys. B212, 321 (1983).

[2] M. Bucher, A. S. Goldhaber, and N. Turok, Phys. Rev. D 52, 3314 (1995); M. Bucher and N. Turok, ibid. 52, 5538 (1995); K. Yamamoto, M. Sasaki, and T. Tanaka, Astrophys. J. 455, 412 (1995).

[3] A. D. Linde, Phys. Lett. B 351, 99 (1995); A. D. Linde and A. Mezhlumian, Phys. Rev. D 52, 6789 (1995).

[4] K. Yamammoto, M. Sasaki, and T. Tanaka, Phys. Rev. D 54,
5031 (1996); J. Garriga, ibid. 54, 4764 (1996); J. GarciaBellido, ibid. 54, 2473 (1996); J. D. Cohn, ibid. 54, 7215 (1996).

[5] A. M. Green and A. R. Liddle, Phys. Rev. D 55, 609 (1997); J. Garcia-Bellido and A. D. Linde, ibid. 55, 7480 (1997).

[6] M. Sasaki and T. Tanaka, Phys. Rev. D 54, 4705 (1996); in Proceedings of the Sixth Workshop on General Relativity and Gravitation, Tokyo, 1996 (unpublished).

[7] J. Garcia-Bellido and A. R. Liddle, Phys. Rev. D 55, 4603 (1997). 